\title{
On the Feasibility of Interference Alignment in the Cellular Network
}

\author{
Hua Chen ${ }^{1}$, Shan $\mathrm{Wu}^{2}$, Ping $\mathrm{Hu}^{1}$ and Zhudi $\mathrm{Xu}^{3}$ \\ ${ }^{1}$ College of Mathematics and Computer Science, Wuhan Textile University, \\ Wuhan, Hubei 430073 - China \\ [e-mail: chenhua@wtu.edu.cn, may2001@163.com] \\ ${ }^{2}$ Department of Public Basic Course, Wuhan Technology and Business University, \\ Wuhan,Hubei 430073 - China \\ [e-mail: hariny@163.com] \\ ${ }^{3}$ School of Electronic Information and Communication, Huazhong University of Sci. \& Tech., \\ Wuhan,Hubei 430074 - China \\ [e-mail: zhudixu@163.com] \\ *Corresponding author: Shan Wu and Zhudi Xu
}

Received February 22, 2017; revised April 27, 2017; revised May 20, 2017; accepted June 6, 2017;

published November 30, 2017

\begin{abstract}
In this paper, we investigate the feasibility of interference alignment(IA) in signal space in the scenario of multiple cell and multiple user cellular networks, as the feasibility issue is closely related to the solvability of a multivariate polynomial system, we give the mathematical analysis to support the constraint condition obtained from the polynomial equations with the tools of algebraic geometry, and a new distribute IA algorithm is also provided to verify the accessibility of the constraint condition for symmetric system in this paper. Simulation results illustrate that the accessibility of the constraint condition is hold if and only if the degree of freedom(DoF) of each user can be divided by both the transmit and receive antenna numbers.
\end{abstract}

Keywords: multiple cell, interference alignment, MIMO, interference broadcast channel 


\section{Introduction}

$\mathbf{W}_{\text {ith the exponential increasing data service requirement, future wirelss system faces great }}$ challenge for massive data transmission, high density wireless network will be deployed widely, in which interference will severely affect the high speed wireless transmission, interference management becomes the bottleneck for 5G high density wireless network. Traditional interference management strategies including frequency division multiplexing and sector classification can not make good use of spectrum resources, therefore it is very urgent to study high efficient interference management technologies for the future wireless networks.

In order to improve the spectrum efficiency, Jafar et al propose the interference alignment(IA) schemes[1,2], which puts interference into a space whose degree of dimensions is as least as possible and leave more dimensions for transmitting useful signal by designing clever precoding matrices or beamforming vectors. And in this way, the desired signals to the receivers can be easily distinguished from the interference. By using IA schemes, Cadambe and Jafar has found a very surprising result for K user interference channels[3] , which claims that a $K / 2$ sum degree of freedoms(DoF) can be achieved in the scenario of the infinite channel diversity length. In contrast, the traditional schemes such as TDMA or the strategy treating interference as the background noise can only achieve a sum DOF of 0 and 1 . After that work, Jafar discussed the feasibility of IA in MIMO interference networks, of which he put forward a necessary condition concerned with the signal space dimensions[4]. Luo later refined Jafar's work in [5], where he found the accurate necessary conditions for the feasibility of IA in MIMO interference networks in signal space and gave a rigorously proof for it with the tools of algebraic geometry[6,7]. However, these results are all concerned with MIMO interference channels. For cellular network, Sun et al developed a tight outbound of the DOF per cell and provided a achievable scheme exploiting the idea of IA in the signal scale[8]. To achieve the upper bound of DOF per cell, the coding dimension and the SNR of the IA scheme proposed in their paper are both infinite, which make it of little use in practical scenes. On the other hand, the beamforming-based IA technology is found to be satisfactory for practical scenarios where the SNR is finite, and it also incorporates the advantages of analytical tractability and insenstivity to practical limitations. However, the problem of the feasibility for IA based on signal space in cellular networks is still unsolved. We think this problem is meaningful for its potential being extended to the practical fields such as user selection problem in multi-cell case and so on. And all of these inspire us to search for the constraint condition for IA only based on beamforming in cellular networks.

The motivation of this paper is to explore IA scheme condition in cellular networks when the channel diversity is limited,which is very useful for the practical use. More specifically, our goal can be rephrased as following: finding the constraint conditions for the feasibility of IA in the cellular network only based on the signal vector space and designing an interference alignment algorithm satisfying the proposed IA scheme conditions.

The rest of this paper is organized as follows:Section2 describes the interference model for cellular network, Section 3 provides the mathematical conjecture and proof of the constraint conditions for interference alignment in cellular networks. Section 4 provides a simple interference alignment algorithm to support our theoretical analysis and section 5 gives the simulation results of this algorithm. At last, we will provide some concluding remarks. 


\section{System Model}

Consider a cellular network which contains several cells. users only communicate with their desired base station in each cell. Each user and base station are equipped with multiple antennas. The two cell scenario is illustrated in Fig. 1.

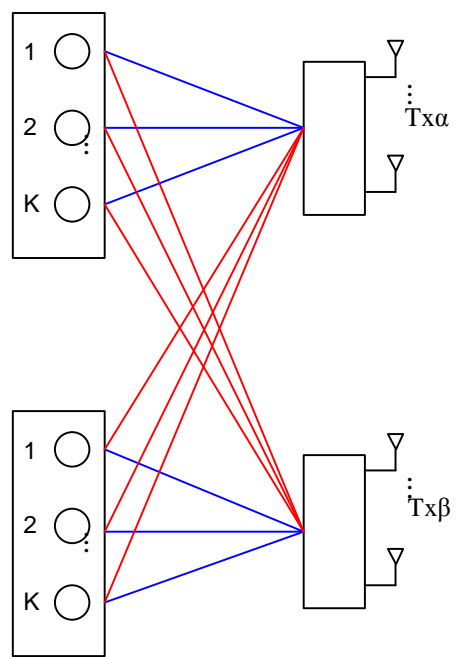

Fig. 1. two-cell interference cellular network

The interference of each user at the base station contains two parts: the interference from other users in the same cell and the interference from other cells. Consider a $G$-cell cellular network, the base station in cell $i$ is equipped with $M_{i}$ antennas and the user $k$ in cell $i$ is equipped with $N_{i_{k}}$ antennas. The received signal of each user can be expressed as:

$$
\mathbf{y}_{i_{k}}=\mathbf{u}_{i_{k}}^{H} \mathbf{H}_{i_{k}, i} \mathbf{V}_{i} \mathbf{x}_{i}+\sum_{j=1, j \neq i}^{G} \mathbf{u}_{i_{k}}^{H} \mathbf{H}_{i_{k}, j} \mathbf{V}_{j} \mathbf{x}_{j}+\mathbf{u}_{i_{k}}^{H} \mathbf{z}_{i_{k}}
$$

where $\mathbf{u}_{i_{k}} \in \mathbb{C}^{N_{i_{k}} \times d_{i_{k}}}, d_{i_{k}}$ is the DOF of the user $k$ in cell $i, \mathbf{V}_{i}=\left[\begin{array}{llll}\mathbf{v}_{i_{1}} & \mathbf{v}_{i_{2}} & \cdots & \mathbf{v}_{i_{K_{i}}}\end{array}\right]$ is the transmitting vector of the base station in cell $i, \mathbf{v}_{i_{k}} \in \mathbb{C}^{M_{i_{k}} \times d_{i_{k}}}, \mathbf{H}_{i_{k}, j} \in \mathbb{C}^{N_{i_{k}} \times M_{i_{k}}}$ is the channel matrix from the base station in cell $j$ to the user $k$ in cell $i, z_{i_{k}} \in \mathbb{C}^{N_{i_{k}} \times 1}$ is the additive white Gaussian noise vector with zero-mean and covariance matrix $\sigma_{n}^{2} \mathbf{I}, \mathbf{x}_{i}=\left[\begin{array}{llll}x_{i 1} & x_{i 2} & \cdots & x_{i_{K_{i}}}\end{array}\right]^{T}$ is the signals from user one to user $\mathrm{K}$ in cell $i$. The first part of the expressions consists of the desired signal and the intra-cell interference from other users, the second part is the interference from other cells.

\section{Constraint Conditions for Linear IA Schemes}

Several theoretical results about the feasibility of linear interference alignment in cellular networks will be revealed in this part. The idea of "proper system " for the feasibility of IA schemes in K-user MIMO interference channel based only on beamforming is proposed by Jafar at first[4]. Then, Luo established a general condition that must be satisfied for linear 
interference alignment schemes in MIMO interference channels as well as a theoretical proof with the tools of algebraic geometry[7].

We can adress this problem for the multi-cell case in the same way as in the $K$-user MIMO interference channel. First of all, relate the feasibility issue to the solvability of a multivariate polynomial system. The multivariate polynomial system in a cellular network can be expressed as follows:

$$
\left\{\begin{array}{c}
\operatorname{rank}\left(\mathbf{u}_{i_{k}}^{H} \mathbf{H}_{i_{k}, i} \mathbf{v}_{i_{k}}\right)=d_{i_{k}} \\
\mathbf{u}_{i_{k}}^{H} \mathbf{H}_{i_{k}, i} \mathbf{v}_{i_{l}}=0 \\
\mathbf{u}_{i_{k}}^{H} \mathbf{H}_{i_{k}, j} \mathbf{v}_{j_{k}}=0
\end{array}\right.
$$

where $i, j \in[1, G], j \neq i, k, l \in\left[1, K_{i}\right], l \neq k$, it means that the dimension of the useful signal is equal to the DOF of each user and all the interference will be aligned to the space orthogonal to the useful signal space. As we have addressed before, the interference contains two parts :the interference from other users in the same cell and the interference from other cells. By studying the solvability of the polynomial system above carefully, we can derive an important result:

$$
\sum_{i=1}^{G} \sum_{k=1}^{K_{i}}\left(M_{i}+N_{i_{k}}-2 d_{i_{k}}\right) d_{i_{k}} \geq \sum_{i=1}^{G} \sum_{k=1}^{K_{i}} d_{i_{k}}\left(\sum_{j=1, j \neq i}^{G} \sum_{l=1}^{K_{j}} d_{j_{l}}+\sum_{l=1, l \neq k}^{K_{i}} d_{i_{l}}\right)
$$

Eq.(3) gives the constraint the DoF should satisfy concerned with the antenna configuration.

The proof process of this theorem is similar to the case for the K user MIMO interference channel. For the ease of analysis, we will introduce some basic concepts for algebraic geometry theory in the following part.

Define two field $\mathcal{K}$ and $\mathcal{F}$,where $\mathcal{K} \subseteq \mathcal{F}, \mathcal{F}$ is an extension of $\mathcal{K}$. For $\alpha_{1}, \alpha_{2}, \cdots, \alpha_{n} \in \mathcal{F}$, if there exists a nonzero polynomial $f$ such that $f\left(\alpha_{1}, \alpha_{2}, \cdots, \alpha_{n}\right)=0$, then ,we say $\alpha_{1}, \alpha_{2}, \cdots, \alpha_{n}$ are algebraic dependent with each other. $f \in \mathcal{K}\left(z_{1}, z_{2}, \cdots, z_{n}\right)$, $\mathcal{K}\left(z_{1}, z_{2}, \cdots, z_{n}\right)$ represents the ring of polynomials with coefficients draw from $\mathcal{K}$. If the nonzero polynomial $f$ doesn't exist, then we say that $z_{1}, z_{2}, \cdots, z_{n}$ are algebraic independent. Also, we define the transcendence degree of $\mathcal{F}$ over $\mathcal{K}$ as the largest cardinality of an algebraically independent set. Similar to the characteristics of the linear independence , a important result holds for algebraic independence:

Assume $g_{1}, g_{2}, \cdots, g_{n+1}$ are $n+1$ functions defined on $n$ variables $x_{1}, x_{2}, \cdots, x_{n}$, then $g_{1}, g_{2}, \cdots, g_{n+1}$ must be algebraic dependent, and there must exist a nonzero polynomial $f$ such that:

$$
f\left(g_{1}, g_{2}, \cdots g_{n+1}\right)=0, \quad \forall\left(x_{1}, x_{2}, \cdots, x_{n}\right)
$$

This theorem will be used for the following proof. We can rewrite the receive and transmit vectors by using the linear transformation as the following forms:

$$
\mathbf{u}_{i_{k}}=\mathbf{P}_{i_{k}}^{u}\left[\begin{array}{c}
\mathbf{I} \\
\overline{\mathbf{u}}_{i_{k}}
\end{array}\right] \mathbf{Q}_{i_{k}}^{u}, \quad \mathbf{v}_{i_{k}}=\mathbf{P}_{i_{k}}^{v}\left[\begin{array}{c}
\mathbf{I} \\
\overline{\mathbf{v}}_{i_{k}}
\end{array}\right] \mathbf{Q}_{i_{k}}^{v}
$$


where $\overline{\mathbf{u}}_{i_{k}} \in \mathbb{C}^{\left(N_{i_{k}}-d_{i_{k}}\right) \times d_{i_{k}}}, \overline{\mathbf{v}}_{i_{k}} \in \mathbb{C}^{\left(M_{i_{k}}-d_{i_{k}}\right) \times d_{i_{k}}}, \mathbf{P}_{i_{k}}^{u}$ and $\mathbf{P}_{i_{k}}^{v}$ are square permutation matrices of size $N_{i_{k}} \times N_{i_{k}}$ and $M_{i_{k}} \times M_{i_{k}}$ respectively, $\mathbf{Q}_{i_{k}}^{u}$ and $\mathbf{Q}_{i_{k}}^{v}$ are invertible matrices of size $d_{i_{k}} \times d_{i_{k}}$, I is identity matrix. Then we define $\overline{\mathbf{H}}_{i_{k}, j}$ as:

$$
\overline{\mathbf{H}}_{i_{k}, j_{l}}=\mathbf{P}_{i_{k}}^{u} \quad{ }^{-1} \mathbf{H}_{i_{k}, j} \mathbf{P}_{j_{l}}^{v}-1=\left[\begin{array}{ll}
\overline{\mathbf{H}}_{i_{k}, j_{l}}^{(1)} & \overline{\mathbf{H}}_{i_{k}, j_{l}}^{(2)} \\
\overline{\mathbf{H}}_{i_{k}, j_{l}}^{(3)} & \overline{\mathbf{H}}_{i_{k}, j_{l}}^{(4)}
\end{array}\right]
$$

$\overline{\mathbf{H}}_{i_{k}, j_{l}}$ has been partitioned into four parts, where $\overline{\mathbf{H}}_{i_{k}, j_{l}}^{(1)} \in \mathbb{C}^{d_{i_{k}} \times d_{j l}}$ and $i, j=1,2, \cdots G, k=1,2, \cdots, K_{i}, l=1,2, \cdots, K_{j}$ when $i=j, k \neq l$. Using (5) and (6), the feasibility conditions (2) can be rewritten as

Or

$$
\left[\begin{array}{ll}
\mathbf{I} & \overline{\mathbf{u}}_{i_{k}}^{H}
\end{array}\right]\left[\begin{array}{cc}
\overline{\mathbf{H}}_{i_{k}, j_{l}}^{(1)} & \overline{\mathbf{H}}_{i_{k}, j_{l}}^{(2)} \\
\overline{\mathbf{H}}_{i_{k}, j_{l}}^{(3)} & \overline{\mathbf{H}}_{i_{k}, j_{l}}^{(4)}
\end{array}\right]\left[\begin{array}{c}
\mathbf{I} \\
\overline{\mathbf{v}}_{j_{l}}^{H}
\end{array}\right]=\mathbf{0}
$$

$$
\overline{\mathbf{H}}_{i_{k}, j_{l}}^{(1)}+\overline{\mathbf{u}}_{i_{k}}^{H} \overline{\mathbf{H}}_{i_{k}, j_{l}}^{(3)}+\overline{\mathbf{H}}_{i_{k}, j_{l}}^{(2)} \overline{\mathbf{v}}_{j_{l}}^{H}+\overline{\mathbf{u}}_{i_{k}}^{H} \overline{\mathbf{H}}_{i_{k}, j_{l}}^{(4)} \overline{\mathbf{v}}_{j_{l}}^{H}=0
$$

When $i=j, k \neq l$. The total number of equations in (8) is :

$$
\sum_{i=1}^{G} \sum_{k=1}^{K_{i}} d_{i_{k}}\left(\sum_{j=1, j \neq i}^{G} \sum_{l=1}^{K_{j}} d_{j_{l}}+\sum_{l=1, l \neq k}^{K_{i}} d_{i_{l}}\right)
$$

and the total number of unknown variables is :

$$
\sum_{i=1}^{G} \sum_{k=1}^{K_{i}}\left(M_{i}+N_{i_{k}}-2 d_{i_{k}}\right) d_{i_{k}}
$$

Now we manage to prove theorem (3) by contradiction, first we assume the number of variables is smaller than the number of equations:

$$
\sum_{i=1}^{G} \sum_{k=1}^{K_{i}}\left(M_{i}+N_{i_{k}}-2 d_{i_{k}}\right) d_{i_{k}}<\sum_{i=1}^{G} \sum_{k=1}^{K_{i}} d_{i_{k}}\left(\sum_{j=1, j \neq i}^{G} \sum_{l=1}^{K_{j}} d_{j_{l}}+\sum_{l=1, l \neq k}^{K_{i}} d_{i_{l}}\right)
$$

Consider a field $\mathcal{F}$ which is consisting of all rational functions defined on $\overline{\mathbf{u}}_{i_{k}}$ and $\overline{\mathbf{v}}_{i_{k}}$, then the transcendence degree of $\mathcal{F}$ is $\sum_{i=1}^{G} \sum_{k=1}^{K_{i}}\left(M_{i}+N_{i_{k}}-2 d_{i_{k}}\right) d_{i_{k}}$, which is equal to the number of the unknown variables of $\overline{\mathbf{u}}_{i_{k}}$ and $\overline{\mathbf{v}}_{i_{k}}$. Define a matrix $\mathbf{F}_{i_{k}, j_{l}}(\overline{\mathbf{u}}, \overline{\mathbf{v}})$ :

$$
\mathbf{F}_{i_{k}, j_{l}}(\overline{\mathbf{u}}, \overline{\mathbf{v}}) \triangleq-\left(\overline{\mathbf{u}}_{i_{k}}^{H} \overline{\mathbf{H}}_{i_{k}, j_{l}}^{(3)}+\overline{\mathbf{H}}_{i_{k}, j_{l}}^{(2)} \overline{\mathbf{v}}_{j_{l}}^{H}+\overline{\mathbf{u}}_{i_{k}}^{H} \overline{\mathbf{H}}_{i_{k}, j_{l}}^{(4)} \overline{\mathbf{v}}_{j_{l}}^{H}\right)
$$

For all $i, j=1,2, \cdots G, k=1,2, \cdots, K_{i}, l=1,2, \cdots, K_{j}$. When $i=j, k \neq l, \mathbf{F}_{i_{k}, j_{l}} \in \mathbb{C}^{d_{i_{k}} \times d_{j l}}$ and each entry of $\mathbf{F}_{i_{k}, j_{l}}$ is a quadratic polynomial function of the entries in the matrix $\overline{\mathbf{u}}_{i_{k}}$ and $\overline{\mathbf{v}}_{i_{k}}$. The total number of the entries in $\mathbf{F}_{i_{k}, j_{l}}$ is $\sum_{i=1}^{G} \sum_{k=1}^{K_{i}} d_{i_{k}}\left(\sum_{j=1, j \neq i}^{G} \sum_{l=1}^{K_{j}} d_{j_{l}}+\sum_{l=1, l \neq k}^{K_{i}} d_{i_{l}}\right)$, and if the inequality in (9) holds, then, from the theorem in (4), we can know that there must exist a nonzero polynomial $p$ such that: 


$$
p\left(\mathbf{F}_{1_{1}, 1_{2}}(\overline{\mathbf{u}}, \overline{\mathbf{v}}), \mathbf{F}_{1_{1}, 1_{3}}(\overline{\mathbf{u}}, \overline{\mathbf{v}}), \cdots, \mathbf{F}_{G_{K_{G}}, G_{K_{G}-1}}(\overline{\mathbf{u}}, \overline{\mathbf{v}})\right)=0
$$

A useful local expansion of a multivariate polynomial function for matrix will be used in the following analysis:

$$
f(\mathbf{X})=f(\overline{\mathbf{X}})+\sum_{i=1}^{n} \operatorname{Tr}\left(\left(\mathbf{X}_{i}-\overline{\mathbf{X}}_{i}\right) \mathbf{G}_{i}(\mathbf{X})\right), \quad \forall \mathbf{X}
$$

Where each entry of $\mathbf{G}_{i}(\mathbf{X})$ will only depend on the entries of $\overline{\mathbf{X}}$ and the coefficients of function $f$.

By using the expansion (14), in which we have let $\mathbf{X}:=\left(\overline{\mathbf{H}}_{1_{1}, 1_{2}}^{(1)}, \overline{\mathbf{H}}_{1_{1}, 1_{3}}^{(1)}, \cdots, \overline{\mathbf{H}}_{G_{K_{G}}, G_{K_{G}-1}}^{(1)}\right)$ and $\overline{\mathbf{X}}:=\left(\mathbf{F}_{1_{1}, 1_{2}}(\overline{\mathbf{u}}, \overline{\mathbf{v}}), \mathbf{F}_{1_{1}, 1_{3}}(\overline{\mathbf{u}}, \overline{\mathbf{v}}), \cdots, \mathbf{F}_{G_{K_{G}}, G_{K_{G}-1}}(\overline{\mathbf{u}}, \overline{\mathbf{v}})\right)$ and we have:

$$
\begin{aligned}
p\left(\overline{\mathbf{H}}_{1_{1}, 1_{2}}^{(1)}, \overline{\mathbf{H}}_{1_{1}, 1_{3}}^{(1)}, \cdots, \overline{\mathbf{H}}_{G_{K_{G}}, G_{K_{G}-1}}^{(1)}\right)= & p\left(\mathbf{F}_{1_{1}, 1_{2}}(\overline{\mathbf{u}}, \overline{\mathbf{v}}), \mathbf{F}_{1_{1}, 1_{3}}(\overline{\mathbf{u}}, \overline{\mathbf{v}}), \cdots, \mathbf{F}_{G_{K_{G}}, G_{K_{G}-1}}(\overline{\mathbf{u}}, \overline{\mathbf{v}})\right) \\
& +\sum_{i_{k} \neq j_{l}} \operatorname{Tr}\left(\left(\overline{\mathbf{H}}_{i_{k}, j_{l}}^{(1)}-\mathbf{F}_{i_{k}, j_{l}}(\overline{\mathbf{u}}, \overline{\mathbf{v}})\right) \mathbf{Q}_{i_{k}, j_{l}}(\overline{\mathbf{u}}, \overline{\mathbf{v}})\right)
\end{aligned}
$$

Where $\mathbf{Q}_{i_{k}, j_{l}}(\overline{\mathbf{u}}, \overline{\mathbf{v}})$ is a matrix with the size $d_{i_{k}} \times d_{j_{l}}$ Then, from (13), we can obtain

$$
p\left(\overline{\mathbf{H}}_{1_{1}, 1_{2}}^{(1)}, \overline{\mathbf{H}}_{1_{1}, 1_{3}}^{(1)}, \cdots, \overline{\mathbf{H}}_{G_{K_{G}}, G_{K_{G}-1}}^{(1)}\right)=\sum_{i_{k} \neq j_{l}} \operatorname{Tr}\left(\left(\overline{\mathbf{H}}_{i_{k}, j_{l}}^{(1)}-\mathbf{F}_{i_{k}, j_{l}}(\overline{\mathbf{u}}, \overline{\mathbf{v}})\right) \mathbf{Q}_{i_{k}, j_{l}}(\overline{\mathbf{u}}, \overline{\mathbf{v}})\right)
$$

If the alignment condition (8) is hold , i.e., $\overline{\mathbf{H}}_{i_{k}, j_{l}}^{(1)}-\mathbf{F}_{i_{k}, j_{l}}(\overline{\mathbf{u}}, \overline{\mathbf{v}})=0$, then we can obtain

$$
p\left(\overline{\mathbf{H}}_{1_{1}, 1_{2}}^{(1)}, \overline{\mathbf{H}}_{1_{1}, 1_{3}}^{(1)}, \cdots, \overline{\mathbf{H}}_{G_{K_{G}}, G_{K_{G}-1}}^{(1)}\right)=0
$$

The function $p$ is a polynomial independent of the channel matrices $\overline{\mathbf{H}}_{i_{k}, j_{l}}^{(1)}$ whose elements are drawn from a probability distribution. So, the condition (16) will always hold if and only if function $p$ is identically zero. This is contradiction to the condition that $p$ is a nonzero polynomial. Thus, we know that the assumption $\sum_{i=1}^{G} \sum_{k=1}^{K_{i}}\left(M_{i}+N_{i_{k}}-2 d_{i_{k}}\right) d_{i_{k}}<\sum_{i=1}^{G} \sum_{k=1}^{K_{i}} d_{i_{k}}\left(\sum_{j=1, j \neq i}^{G} \sum_{l=1}^{K_{j}} d_{j_{l}}+\sum_{l=1, l \neq k}^{K_{i}} d_{i_{l}}\right)$ is not true, which proves the theorem in (3).

\section{IA Algorithm for Celluar Networks}

In last section, a constraint condition has been found for linear IA in cellular networks: $\sum_{i=1}^{G} \sum_{k=1}^{K_{i}}\left(M_{i}+N_{i_{k}}-2 d_{i_{k}}\right) d_{i_{k}} \geq \sum_{i=1}^{G} \sum_{k=1}^{K_{i}} d_{i_{k}}\left(\sum_{j=1, j \neq i}^{G} \sum_{l=1}^{K_{j}} d_{j_{l}}+\sum_{l=1, l \neq k}^{K_{i}} d_{i_{l}}\right)$. However, the question that whether the theorem in (3) is tight or not, especially in some special cases, need to be further resolved. Consider the special case where the number of users in each cell is $K$, the DoF of each user is $d$, each base station equips with $M$ transmit antennas and each user equips with $N$ receive antennas. Assume that $M$ and $N$ can be divided by $d$, then theorem (3) can be written as a simple inequality: 


$$
M+N \geq(G K+1) d
$$

It's hard to construct a general solution of that special case when the equality is hold in our theorem. So, we will put forward an IA algorithm to check and verify our result . In our algorithm, we use the receive matrix $\mathbf{u}_{i_{k}}$ to eliminate the interference from other cells. The transmit matrix $\mathbf{v}_{i_{k}}$ will eliminate the interference from other users in the same cell and part of the interference from other cells.

[11] has put forward an IA algorithm for MIMO interference network, which chooses the eigenvector corresponding to the minimum eigenvalues of the total interference as the receive and transmit matrix. We can find our receive matrix $\mathbf{u}_{i_{k}}$ with the similar method. More specifically, we choose the eigenvector corresponding to the minimum eigenvalues of the total interference from other cells to be the receive matrix $\mathbf{u}_{i_{k}}$. The total interference from other cells at the receiving terminal of user $k$ in cell $i$ can be represented as:

$$
\mathbf{Q}_{i_{k}}=\sum_{j=1, j \neq i}^{G} \sum_{l=1}^{K} \mathbf{H}_{i_{k}, j} \mathbf{v}_{j_{l}}\left(\mathbf{v}_{j_{l}}\right)^{\dagger}\left(\mathbf{H}_{i_{k}, j}\right)^{\dagger}
$$

where $i=1,2, \cdots G, k=1,2, \cdots, K$ and our receiving terminal will choose the eigenvector corresponding to the minimum eigenvalues $\lambda_{i_{k}}^{\min }$ of $\mathbf{Q}_{i_{k}}$ to be the receive matrix $\mathbf{u}_{i_{k}}$, so we have $\mathbf{Q}_{i_{k}} \mathbf{u}_{i_{k}}=\lambda_{i_{k}}^{\min } \mathbf{u}_{i_{k}}$. Assume that $\mathbf{I}_{i_{k}}$ represents the power of the interference from other cells and we have:

$$
\mathbf{I}_{i_{k}}=\mathrm{T}_{\mathrm{r}}\left[\left(\mathbf{u}_{i_{k}}\right)^{\dagger} \mathbf{Q}_{i_{k}} \mathbf{u}_{i_{k}}\right]=\mathrm{T}_{\mathrm{r}}\left[\left(\mathbf{u}_{i_{k}}\right)^{\dagger} \lambda_{i_{k}}^{\min } \mathbf{u}_{i_{k}}\right]=\lambda_{i_{k}}^{\min }
$$

From (17), we can see that the power of the interference from other cells depend only on $\lambda_{i_{k}}^{\min }$. And when $\lambda_{i_{k}}^{\min }$ is zero, the whole interference of other cells will be totally eliminated.

The receive matrix will remove part of the interference from other cells and the transmit matrix is designed to eliminate the remaining interference including all the interference from other users in the same cell and part of the interference from other cells. MMSE method can be used to find transmit matrix $\mathbf{v}_{i_{k}}$ ([12]-[14]):

$$
\mathbf{v}_{i_{k}}=\left(\sum_{j=1}^{G} \sum_{l=1}^{K}\left(\mathbf{H}_{i_{k}, j}\right)^{\dagger} \mathbf{u}_{j_{l}}\left(\mathbf{u}_{j_{l}}\right)^{\dagger} \mathbf{H}_{i_{k}, j}+\rho \sigma_{n}^{2} \mathbf{I}\right)^{-1}\left(\mathbf{H}_{i_{k}, i}\right)^{\dagger} \mathbf{u}_{i_{k}}
$$

Where $\sigma_{n}^{2}$ is the power of noise and $\mathbf{I} \in \mathbb{C}^{M \times M}$ is the identity matrix.. It is worthy to note that the MMSE method is a global general algorithm, of which the zero forcing algorithm and the maximum SLNR algorithm are the special case of $\rho \rightarrow 0$ and $\rho \rightarrow 1$ respectively. The detailed IA algorithm is as follows:

1) Initialize the transmit matrices $\mathbf{v}_{i_{k}}$

2) Compute the interference comeing from other cells of each user:

$$
\mathbf{Q}_{i_{k}}=\sum_{j=1, j \neq i}^{G} \sum_{l=1}^{K} \mathbf{H}_{i_{k}, j} \mathbf{v}_{j_{l}}\left(\mathbf{v}_{j_{l}}\right)^{\dagger}\left(\mathbf{H}_{i_{k}, j}\right)^{\dagger}
$$


3) Obtain the receive matrices $\mathbf{u}_{i_{k}}$ from $\mathbf{Q}_{i_{k}}: \mathbf{u}_{i_{k}}=$ min_eigenvecor $\left(\mathbf{Q}_{i_{k}}\right)$, $\mathbf{u}_{i_{k}}$ is the eigenvector corresponding to the minimum eigenvalue of $\mathbf{Q}_{i_{k}}$

4) Compute $\mathbf{v}_{i_{k}}$ from $\mathbf{u}_{i_{k}}$ :

$$
\mathbf{v}_{i_{k}}=\left(\sum_{j=1}^{G} \sum_{l=1}^{K}\left(\mathbf{H}_{i_{k}, j}\right)^{\dagger} \mathbf{u}_{j_{l}}\left(\mathbf{u}_{j_{l}}\right)^{\dagger} \mathbf{H}_{i_{k}, j}+\rho \sigma_{n}^{2} \mathbf{I}\right)^{-1}\left(\mathbf{H}_{i_{k}, i}\right)^{\dagger} \mathbf{u}_{i_{k}}
$$

5) Repeat step 2) until the algorithm converges

\section{Experimental Classification Results and Analysis}

In this section, we will verify our analysis via simulation and numerical results. Assume that the antenna number of each base station is $M$, the antenna number of each user is $N$ and the user number in each cell is $K$, Table $\mathbf{1}$ lists the simulation paramenters

Table 1. parameter list

$\begin{array}{cc}\text { System parameters } & \text { Symbol } \\ \text { Num of cells } & G \\ \text { Bas antenna num } & M \\ \text { User antenna num } & N \\ \text { Num of users per cell } & K \\ \text { DOF of each user } & d\end{array}$

Fig. 2 demonstrates the progression of the algorithm when the number of users $\mathrm{K}$ is at the threshold $(K=5)$, in which number of cells $G=2$, number of $B S$ antennas $M=9$, number of user's antennas $N=3$, the degree of freedom of each user $d=1$. From this result, we can conclude that this algorithm converges fast and can put the interference to zero when $\mathrm{K}$ is at the threshold.

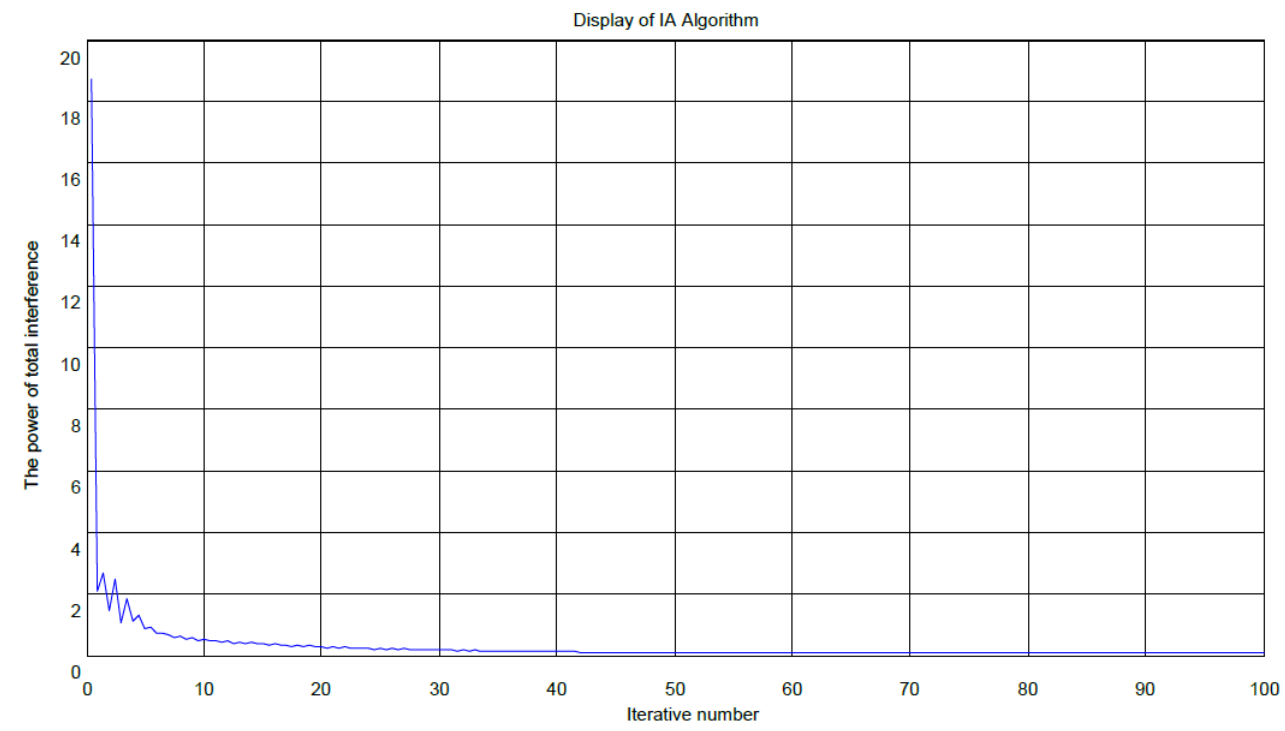

Fig. 2. Plot demonstrating the progression of the algorithm ( $G=2, K$ is set as the threshold value ) 
Fig. 3 displays the progression of the algorithm when the number of users $\mathrm{K}$ is less than the threshold ( $\mathrm{K}=4$ ), and the other parameters are the same as the previous simulation. We can see, when $\mathrm{K}$ is beneath the threshold value, our algorithm converges fairly fast and stable and puts the interference to zero.

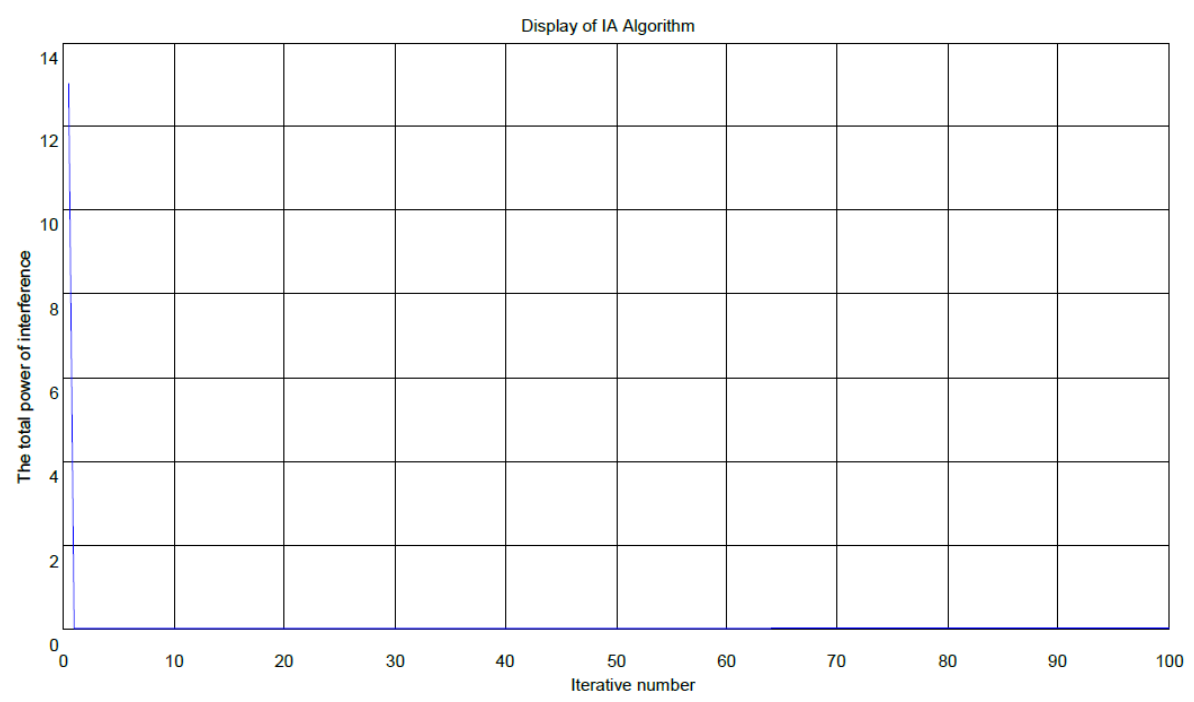

Fig. 3. Plot demonstrating the progression of the algorithm ( $\mathrm{G}=2 \mathrm{~K}$ is less than the threshold value ).

Fig. 4 displays the progression of the algorithm when number of users $\mathrm{K}$ is greater than the threshold $(K=6)$, while the other parameters are the same as previous simulations. We can find that our algorithm can converge but the interference cannot be forced to zero when $\mathrm{K}$ is above the threshold.

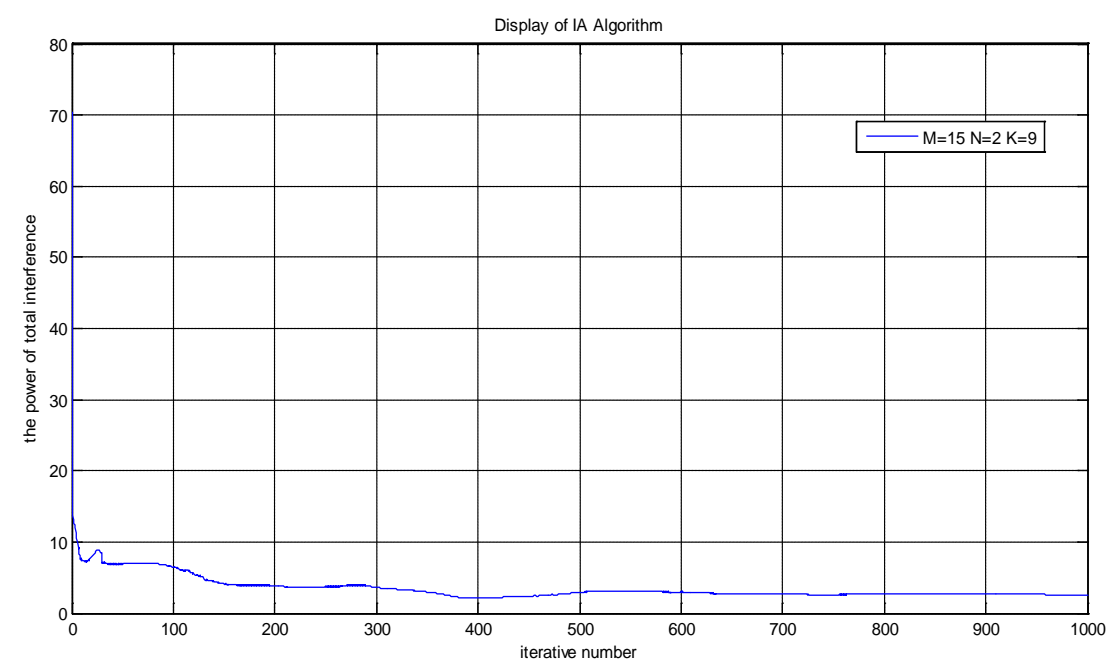

Fig. 4. Plot demonstrating the progression of the algorithm(G=2, $\mathrm{K}$ is more than the threshold value) 
Fig. $2 \sim$ Fig. 4 illustrate the progressions of the algorithm when number of users $\mathrm{K}$ is equal to, less than and greater than the threshold respectively. In conclusion, when $\mathrm{K}$ is less than the threshold, the algorithm converges after several iterations, when $\mathrm{K}$ is equal to or greater than the threshold, the convergence speed is slower; On the other hand, when $\mathrm{K}$ is less than or equal to the threshold, the interference can be forced to zero, while the interference level would be converge to a non-zero value when $\mathrm{K}$ is greater than the threshold.

Fig. 5 compares the performance of the two algorithms. In multi-cell interference alignment algorithm, the system throughput increases almost linearly with the SNR, while in interference zero-forced algorithm, the system throughput is trending to a flat level with the SNR increasing, which is because the inter-cell interference is limiting the growth of the system capacity badly.

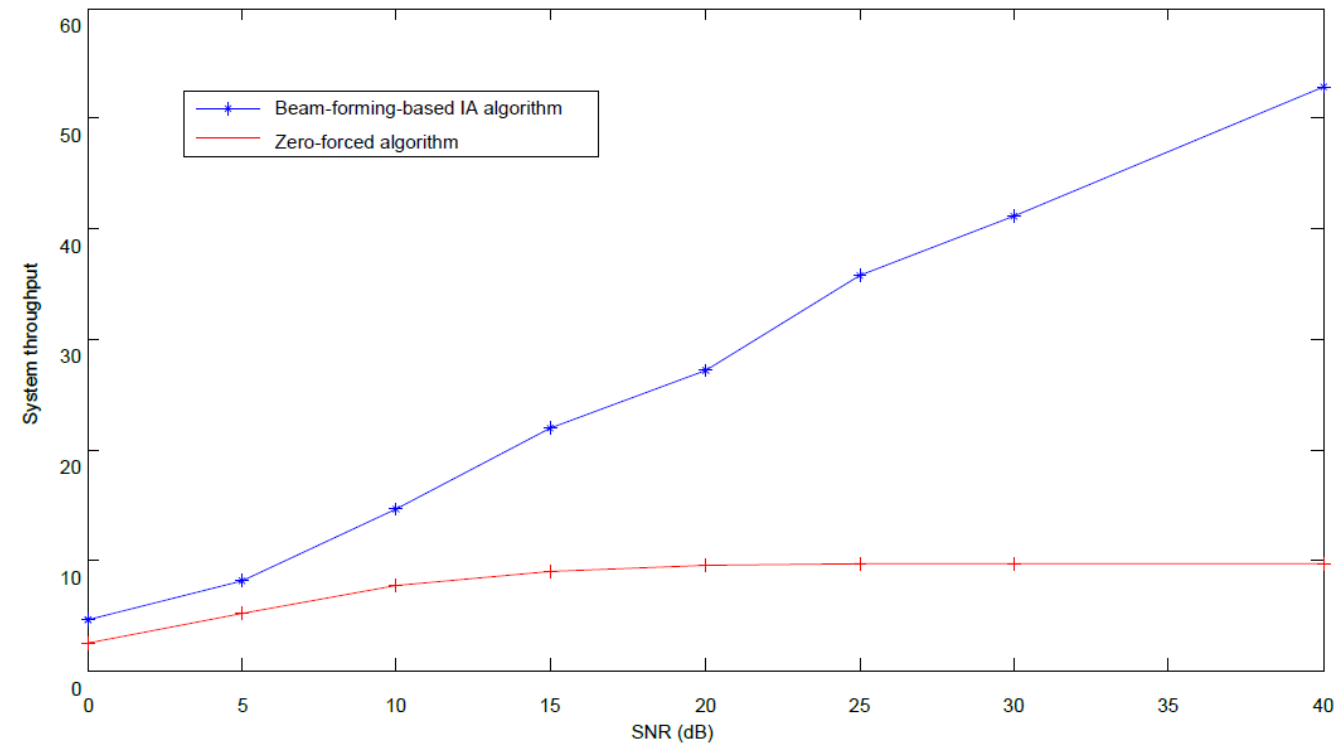

Fig. 5. Plot comparing the performance of algorithms

Table 2. The power of interference and effective signal when $\mathrm{K}$ is set as the threshold

\begin{tabular}{|c|c|c|c|}
\hline System parameters & \multicolumn{3}{|c|}{$\mathrm{M}=5, \mathrm{~N}=2, \mathrm{~K}=3$} \\
\hline \multirow{2}{*}{$\alpha$ cell } & $\begin{array}{c}\text { Interference power } \\
\text { I_10 }\end{array}$ & $\begin{array}{c}\text { Effective signal power } \\
\mathrm{P}\end{array}$ & Ratio P/I_10 \\
\hline User 1 & 3.08 & 1.05 & 3.42 \\
\hline User 2 & 4.88 & 1.63 & 3.34 \\
\hline User 3 & 4.66 & 1.59 & 3.41 \\
\hline \multirow{2}{*}{$\beta$ cell } & Interference power & Effective signal power & Ratio P/I_10 $0^{3}$ \\
\hline User 1 & I_10 & $\mathrm{P}$ & 13.3 \\
\hline User 2 & 0.84 & 1.12 & 2.96 \\
\hline User 3 & 4.01 & 1.19 & 1.76 \\
\hline
\end{tabular}


Table 3 provides each user's power of interference and effective signal after the algorithm converges, under the condition of two cells and number of users $K$ above the threshold $\frac{M+N-1}{2}$ (Note: the order of magnitude of user's effective signal power $\mathrm{P}$ is 1 and the order of magnitude of interference power $\mathrm{I}$ is 0.1 . ). In this scenario, $\mathrm{K}$ is only larger than $\frac{M+N-1}{2}$ by 1 , but each user's ratio of effective signal power to interference power is down to about 4, which is far from the 2000 when $\mathrm{K}$ is set as the threshold. It means that the interference cannot be cancelled, which proves numerically that $\frac{M+N-1}{2}$ is the theoretic maximum of number of users $\mathrm{K}$.

Table 3. The power of interference and effective signal when $\mathrm{K}$ is larger than the threshold

\begin{tabular}{|c|c|c|c|}
\hline System parameters & \multicolumn{3}{|c|}{$\mathrm{M}=5, \mathrm{~N}=2, \mathrm{~K}=3$} \\
\hline \multirow{2}{*}{$\alpha$ cell } & Interference power I & Effective signal power P & Ratio P/I \\
\hline User 1 & 0.57 & 1.08 & 1.89 \\
\hline User 2 & 0.20 & 1.16 & 5.79 \\
\hline User 3 & 0.25 & 1.22 & 4.85 \\
\hline User 4 & 0.19 & 1.02 & Ratio P/I \\
\hline \multirow{2}{*}{$\beta$ cell } & Interference power I & Effective signal power P & 6.28 \\
\hline User 1 & 0.17 & 1.06 & 4.70 \\
\hline User 2 & 0.16 & 0.77 & 4.24 \\
\hline User 3 & 0.14 & 0.61 & 2.18 \\
\hline User 4 & 0.70 & 1.53 & \\
\hline
\end{tabular}

Table 4 lists the ratio of effective signal power to interference power under different (M, N, K) parameters in two-cell scenario. The signal-to-interference-and-noise-ratio (SINR) is at least 20 when $\mathrm{K}$ is the threshold; while the interference level is as the same order as the signal level when $K$ is larger than the threshold by 1 . When $K$ is set as the threshold, and $M=15, N=2,4$, 6 respectively, the SINR is not higher than other scenarios, and it is hard for the algorithm to search out the sole optimal solution. But when we compared the previous results to the results of scenarios where $\mathrm{K}$ is equal to threshold minus 1 and threshold plus 1 , it is quite easy for us to conclude that $\frac{M+N-1}{2}$ is indeed the threshold that the system can achieve a complete interference alignment.

Table 4. The results with different (M, N, K)

\begin{tabular}{|c|c|c|c|}
\hline \multicolumn{4}{|c|}{ The average of effective signal power P/interference power I } \\
\hline \multirow{2}{*}{$\begin{array}{c}\text { K is threshold } \\
(\mathrm{M}, \mathrm{N}, \mathrm{K})\end{array}$} & $(5,2,3)$ & $(5,4,4)$ & $(5,6,5)$ \\
\cline { 2 - 4 } & $3.27 \times 10^{3}$ & $1.17 \times 10^{4}$ & $9.80 \times 10^{4}$ \\
\hline \multirow{2}{*}{$\begin{array}{c}\mathrm{K}>\text { threshold } \\
(\mathrm{M}, \mathrm{N}, \mathrm{K})\end{array}$} & $(5,2,4)$ & $(5,4,5)$ & $(5,6,6)$ \\
\hline K is threshold & 3.53 & 3.76 & 3.09 \\
\cline { 2 - 4 }$(\mathrm{M}, \mathrm{N}, \mathrm{K})$ & $(10,2,5)$ & $(10,4,6)$ & $(10,6,7)$ \\
\cline { 2 - 4 } & $5.78 \times 10^{3}$ & $1.27 \times 10^{4}$ & $1.63 \times 10^{4}$ \\
\hline
\end{tabular}




\begin{tabular}{|c|c|c|c|}
\hline \multirow{2}{*}{$\begin{array}{c}\mathrm{K}>\text { threshold }(\mathrm{M} \text {, } \\
\mathrm{N}, \mathrm{K})\end{array}$} & $(10,2,6)$ & $(10,4,7)$ & $(10,6,8)$ \\
\hline & 7.50 & 12.22 & 13.11 \\
\hline \multirow{2}{*}{$\begin{array}{l}\mathrm{K}<\text { threshold } \\
(\mathrm{M}, \mathrm{N}, \mathrm{K})\end{array}$} & $(15,2,7)$ & $(15,4,8)$ & $(15,6,9)$ \\
\hline & $5.73 \times 10^{4}$ & $1.52 \times 10^{4}$ & $4.15 \times 10^{4}$ \\
\hline \multirow{2}{*}{$\begin{array}{l}\mathrm{K} \text { is threshold } \\
(\mathrm{M}, \mathrm{N}, \mathrm{K})\end{array}$} & $(15,2,8)$ & $(15,4,9)$ & $(15,6,10)$ \\
\hline & 22.88 & 88.92 & 93.04 \\
\hline \multirow{2}{*}{$\begin{array}{c}\mathrm{K}>\text { threshold }(\mathrm{M}, \\
\mathrm{N}, \mathrm{K})\end{array}$} & $(15,2,9)$ & $(15,4,10)$ & $(15,6,11)$ \\
\hline & 5.90 & 11.44 & 13.74 \\
\hline
\end{tabular}

Table 5 provides the simulation results of the multi-cell interference alignment algorithm under different system parameters $(M, N, K, G, d)$. We can find that, when $K$ is greater than the threshold, the interference of each user cannot be forced to zero and the interference level is as the same order as the signal level; when $\mathrm{K}$ is less than or equal to the threshold, the interference level is tremendously lower than the signal level, which to some degree supports our theory derived in the last chapter: $M+N \geq(G K+1) d$. And according to the simulation results, we speculate that, under the assumption of symmetric systems, the equality holds in the constraint when $\mathrm{M}$ and $\mathrm{N}$ can be divided by the number of data streams of each user.

Table 5. The simulation results under different parameters (G, M, N, K, d)

\begin{tabular}{|c|c|c|c|}
\hline $\begin{array}{c}\text { System parameters } \\
(\mathrm{G}, \mathrm{M}, \mathrm{N}, \mathrm{K}, \mathrm{d})\end{array}$ & $\begin{array}{c}\text { Total interference } \\
\text { I_all }\end{array}$ & $\begin{array}{c}\text { Total signal power } \\
\text { P_all }\end{array}$ & $\begin{array}{c}\text { Ratio } \\
\text { P_all/ I_all }\end{array}$ \\
\hline$(2,5,2,3,1)$ & $6.620 \times 10^{-9}$ & 4.534 & $7.350 \times 10^{8}$ \\
\hline$(2,5,2,4,1)$ & 3.024 & 7.7341 & 2.557 \\
\hline$(3,5,2,2,1)$ & $6.121 \times 10^{-6}$ & 7.238 & $1.182 \times 10^{8}$ \\
\hline$(3,5,2,3,1)$ & 5.025 & 12.004 & 2.390 \\
\hline$(2,10,4,3,2)$ & $1.154 \times 10^{-7}$ & 13.073 & $1.133 \times 10^{8}$ \\
\hline$(2,10,4,4,2)$ & 1.680 & 11.443 & 6.813 \\
\hline$(2,11,3,3,2)$ & 0.320 & 11.050 & 34.542 \\
\hline$(2,11,3,4,2)$ & 2.724 & 21.627 & 7.940 \\
\hline
\end{tabular}

\section{Conclusion}

This paper aims at investigating the problem of the feasibility of IA in cellular networks based only on beamforming. By using the tools of algebraic geometry, we have developed some theoretical results about the feasibility of the linear interference alignment in cellular networks. However, the question that whether the outbound is tight or not is still resolved. It's very difficult to construct a general solution achieving the equality in theorem (18), but a simulation scheme proposed in this paper is used to make up for this discount to a certain degree. By observing the simulation results, several interesting conclusions have been obtained: for symmetric systems ( $M, N, K, d$ of each cell are the same), the bound $M+N \geq(G K+1) d$ is tight when $d$ can be divided by $M$ and $N$. 


\section{References}

[1] M. Maddah-Ali, A. Motahari, and A. Khandani, "Communication over X channel: Signalling and multiplexing gain," Univ. California,Berkeley, CA, Tech. Rep., Dec. 2006. Article (CrossRef Link)

[2] S. A. Jafar and S. Shamai (Shitz), "Degrees of freedom region for the MIMO X channel," IEEE Transactions on Information Theory,Vol.54,No. 1,pp.151-170,Jan. 2008. Article (CrossRef Link)

[3] V. R. Cadambe and S. A. Jafar, "Interference alignment and degrees of freedom of the K-user interference channel," IEEE Trans. Inf. Theory, vol. 54, no. 8, pp. 3425-3441, Aug. 2008. Article (CrossRef Link)

[4] C.M. Yetis, T. Gou, S.A. Jafar, and A.H. Kayran "On Feasibility of Interference Alignment in MIMO Interference Networks,” IEEE Trans. on Signal Processing, Vol. 58, pp. 4771-4782, 2010. Article (CrossRef Link)

[5] Meisam Razaviyayn, Gennady Lyubeznik, Zq Luo, "On the degrees of freedom achievable through interference alignment in a MIMO interference channel," IEEE Transactions on signal procrssing, IEEE Transactions on Signal Processing,Vol.60, No.2, pp.812-821, Feb. 2012. Article (CrossRef Link)

[6] H. Matsumura, Commutative Algebra, New York: Benjamin/Cummings,1980. Article (CrossRef Link)

[7] M. F. Atiyah and I. G. Macdonald, Introduction to Commutative Algebra,New York: Westview, 1994. Article (CrossRef Link)

[8] J. Sun, Y. Liu, and G. Zhu, "On the degrees of freedom of the cellular network," in Proc. of IEEE ICC'10, pp. 1-5, May 2010. Article (CrossRef Link)

[9] K. Gomadam, V. Cadambe, and S. Jafar, "A distributed numerical approach to interference alignment and applications to wireless interference networks," IEEE Transactions on Information Theory,Vol.57,No.6, pp.3309-3322, June 2011. Article (CrossRef Link)

[10] T. Liu and C. Yang, "Interference alignment transceiver design for MIMO interference broadcast channels," in Proc. of IEEE WCNC, 2012. Article (CrossRef Link)

[11] W. Shin, N. Lee, J.-B. Lim, C. Shin, and K. Jang, "On the design of interference alignment scheme for two-cell MIMO interfering broadcast channels," IEEE Trans. Wireless Commun., vol. 10, no. 2, pp. 437-442, Feb. 2011. Article (CrossRef Link)

[12] Q. Shi, M. Razaviyayn, Z. Luo, and C. He, "An iteratively weighted MMSE approach to distributed sum-utility maximization for a MIMO interfering broadcast channel," in Proc. of IEEE ICASSP, 2011. Article (CrossRef Link) 


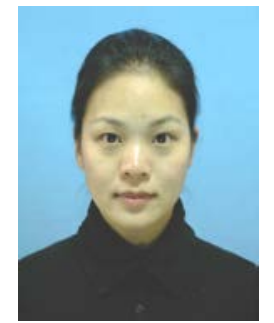

Hua Chen is an associate professor in Wuhan Textile University. Her main research field is sensor network and wireless Communications, include optimization theory and technology, etc. From 2005 to now, her presided several natural science fund projects of Hubei province and several Hubei youth fund projects, published more than 20 papers in the field of sensor network and wireless Communications.

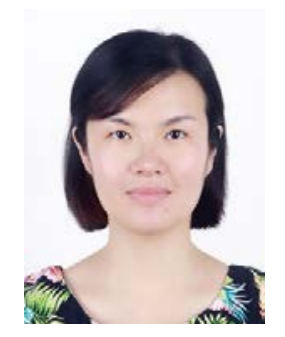

Shan Wu is an Assistant Professor at Wuhan Technology and Business University, Her main research field is mathematical modeling and simulation. She published more than 10 papers in the relevant area.

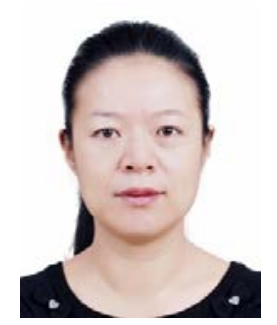

Ping $\mathbf{H u}$ is an associate professor in Wuhan Textile University. Her main research field is Mathematic Model and Risk Theory .From 2004 to now, she took one National natural science fund projects and two Hubei natural science fund projects and , published serveral papers in the field of Mathematic Model and Risk Theory.

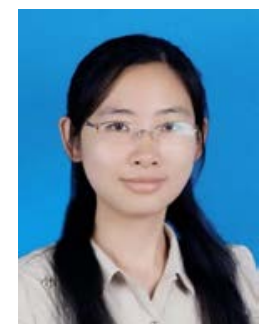

Zhudi Xu graduated from Huazhong University of Science and Tech. in 2014, she is now an engineer of Tencent company, Her main research field is wireless communication. 